\title{
Study of Chaos in the Traffic of Computer Networks
}

\author{
Evgeny Nikulchev \\ Moscow Technological Institute \\ 38A, Leninckiy pr., Moscow, Russia, 119334
}

\author{
Evgeniy Pluzhnik \\ Moscow Technological Institute \\ 38A, Leninckiy pr., Moscow, Russia, 119334
}

\begin{abstract}
Development of telecommunications technology currently determines the growth of research with an aim to find new solutions and innovative approaches to the mathematical description of the processes. One of the directions in the description of traffic in computer networks is focused on studying the properties of chaotic traffic. We offer a complex method for the dynamic chaos determination. It is suggested to introduce additional indicators based on the absence of trivial conservation laws and weak symmetry breaking. The conclusion is made that dynamic chaos in the example of computer network traffic.
\end{abstract}

Keywords-chaos; traffic of computer networks; nonlinear dynamics

\section{INTRODUCTION}

The article is focused on the computation of invariant characteristics of dynamic chaos based on the flow of corporate computer networks.

A significant amount of work on modeling of traffic in computer networks based on queuing theory. This, of course, involves the application of Poisson flow hypothesis, but this hypothesis is often not confirmed by the practice. The hypothesis of the Poisson streams can be used in networks with large redundancy across the width of the channel, in other cases there are other types of distribution and the process requires a fundamentally different approach to modeling.

Today's networks are characterized by the distribution of computing resources and a variety of end-users (from gadgets to appliances that have access to the Internet), with simulation aimed at communication channels control systems creation being a particularly urgent task.

The study specifies distribution which serves as a basis for analyzing data about downloading online channel from the monitoring work of university corporate network, measured in the course of the year. Statistics obtained by removing information from the router interfaces on the number of transmitted data and loading port, protocol snmp, using packet Paessler Router Traffic Grapher, which generates a table with data and graphics load (see Fig. 1).

Empirical histogram frequency channel load is shown in Fig. 2 On the basis of the use of the criteria of fit test and Kolmogorov - Smirnov observed probability distribution is not consistent with a Poisson distribution. Empirical histogram has a "heavy tail", indicating the presence of the peak moments of the network load, in which there is a strong increase in latency and packet loss.

Information on downloading channels was also obtained by monitoring the external communication channels of one of the companies and providers of on-site optimization. The resulting histogram also possesses ponderous tails, indicating the presence of the peak moments of the network load, in which there is a strong increase in delays and loss of information.

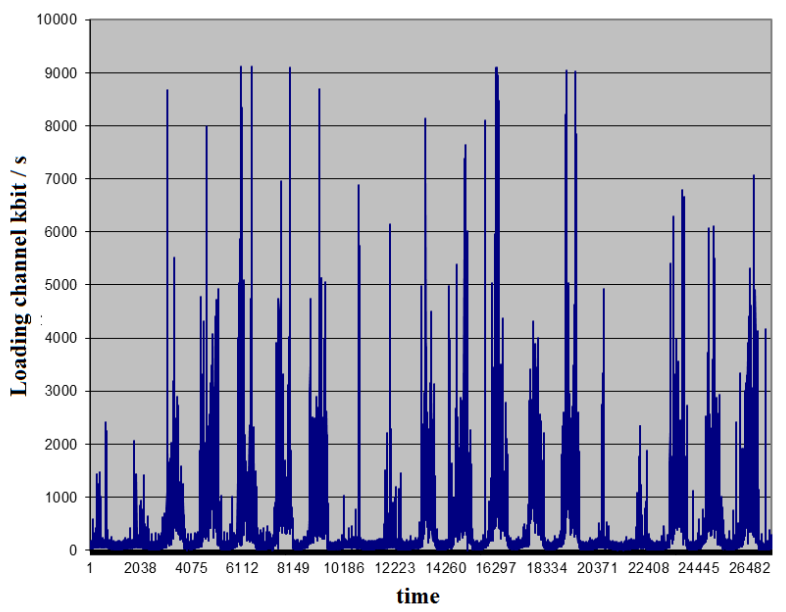

Fig. 1. Fragment of an annual progression loading of the channel network (20 days)

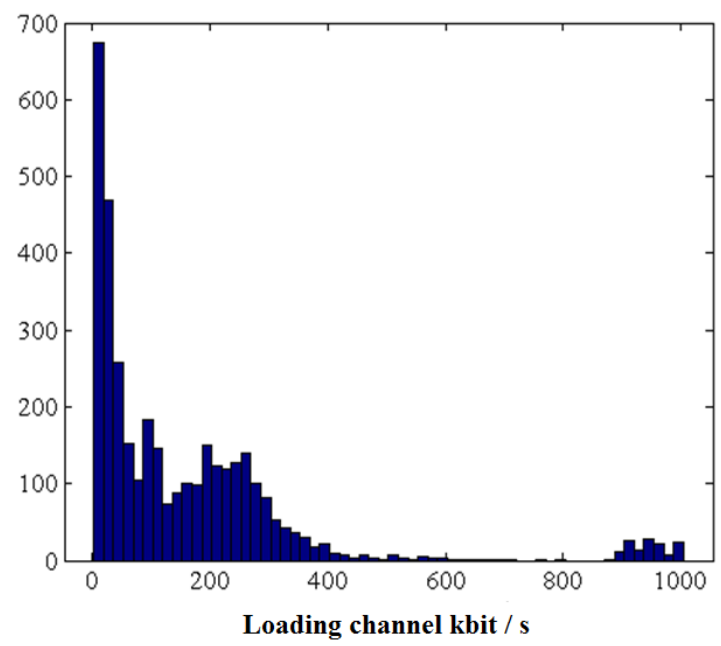

Fig. 2. Empirical histogram loading of the channel network (6 months)

Due to the fact that the distribution function has a heavy tail, and is not consistent with the Poisson distribution, queuing theory for the considered network cannot provide an adequate mathematical description.

As it was noted in [1] for the TCP / IP protocol distribution with ponderous tails makes a major contribution to the self- 
similar nature of the traffic and, consequently, the chaotic nature of the dynamics.

A number of works were focused on the study of chaotic traffic . In [1] the aim is to evaluate the values of the largest Lyapunov exponent on the basis of the traffic generated on the test bench; In [2, 3] Internet traffic is an example for the calculation of various characteristics; In [4] the dynamic properties of the chaos is used to solve telecommunication problems of data exchange, but the study of chaotic properties remained outside publications.

\section{CAlCUlation OF THE Characteristics OF DyNAMiC CHAOS}

It is assumed that the time series generated by the discrete

$$
\mathbf{x}_{k+1}=f\left(\mathbf{x}_{k} \mathbf{x}_{0}\right),
$$

or a continuous system

$$
\frac{d \mathbf{x}(t)}{d t}=F(\mathbf{x}(t), \mathbf{x}(0)) \text {. }
$$

Here, $\mathbf{x}=\left(x_{1}(t), \ldots ., x_{n}(t)\right) ; \mathrm{n}-$ the dimension of the phase space; $t$ - time; $k$ - discrete time (number); $F, f-$ vector function. Phase trajectory of a continuous system is an $\mathrm{n}$-dimensional curve, which is a solution of the system of coordinates of the state space for given initial conditions $\mathbf{x}_{0}$. For discrete systems able to connect lines in accordance with the sequence of samples $\mathrm{k}=1,2, \ldots$

An important concept of dynamical systems is the attractor. For systems in equilibrium, the attractor is a point (with the time change state $\mathrm{x}$ does not change), for oscillatory systems closed paths (cycles). For chaotic systems, there is an attractor, which is called the odd, in this case the trajectories are drawn, but not to the point, a curve, a torus, and in a subset of the phase space. Attractor is an invariant feature of the system, ie. is preserved under a conversion action

Unambiguous characteristics of chaotic signal are a spectrum of Lyapunov exponents. Positive maximum of Lyapunov exponent is a measure of chaotic dynamics, zero maximum Lyapunov exponent denotes a limit cycle or quasiperiodic orbit and negative maximum Lyapunov exponent is a fixed point [2]. System of dimension $\mathrm{n}$ has $\mathrm{n}$ Lyapunov exponents: : $\lambda_{1}, \lambda_{2}, \ldots, \lambda_{n}$, ranked in descending order. According to the definition introduced by Lyapunov:

$$
\lambda_{i}\left(x_{0}\right)=\lim _{t \rightarrow \infty} \frac{1}{t} \ln \frac{\left|\delta_{1}(t)\right|}{\left|\delta_{i}(0)\right|} .
$$

here $\left\{\delta_{i}(t)\right\}$ - the fundamental solution of the system, linearized in the neighborhood of $\mathbf{x}_{0}$.

Dynamical systems, for which the n-dimensional phase volume decreases are called dissipative. If the phase space is conserved, such systems are called conservative. In conservative systems there is always at least one conservation law. The presence of the law of conservation often implies the existence of the corresponding zero Lyapunov exponent. For dissipative dynamical systems sum of Lyapunov exponents is always negative. In dissipative systems, Lyapunov exponents are invariant with respect to all initial conditions.

In terms of Lyapunov, it is possible to provide much information on the observed mode of the dimension of the attractor, if any, and on the entropy of a dynamical system. Dynamic chaos meets the instability of each individual trajectory, ie presence of at least one positive Lyapunov exponent. The attraction of the attractor requires that the phase volumes of large dimensions shrank, then reflected in the Lyapunov spectrum. Knowledge allows us to estimate Lyapunov exponents and the fractal dimension of the attractor [1].

Nevertheless, the number of independent frequencies cannot always find out as zero indicators may be associated with the presence of conserved quantities. The presence of dissipative systems of conservation laws, in general, is not typical, but there are relevant examples.

There is a considerable amount of numerical methods for calculating Lyapunov exponents from time series [2]. It is important that the condition that the number generated by the system under study (1) or (2), a senior figure could be calculated. However, it is impossible to estimate the entire spectrum. For distributed systems, even knowing the system of equations, the evaluation of the Lyapunov exponent is a significant computational complexity.

For the test series, shown in Fig. 1, 2, we calculate the largest Lyapunov exponent. For the calculations we used a system TISEAN. The results of calculations by different methods showed a positive value of the highest exponent.

However, the positivity of the largest Lyapunov exponent cannot be a necessary condition for the existence of chaos. For example, even in a system of Lorentz with positive leading indicator is known in a number of conditions, have a limit cycle.

An additional criterion to use the property of absence of trivial conservation laws was suggested that is - symmetries broadcast, tension and compression. A lowest symmetry violation is used to identify the chaos [5, 6]. Note that the compression phase volume does not mean conversion ratio.

To check the transformation fragment trajectories genetic algorithm and program for MATLAB were developed, their description is given in [3]. At the same time there is a check of the following assumption [6], which proves that the system allows conversion in low-symmetry breaking, i.e. there is some small value, slightly deviating from the symmetric display. Visually, it is geometrically evident at almost similar hinges on the attractor. It is obvious that in such a test, with different initial conditions for systems with regular dynamics is was discovered that they identical symmetry, for more complex but not chaotic - translation (shift of the phase portrait) for systems that tend to stable equilibrium position compression, etc., and for the chaos - almost repeated portions of phase trajectories.

Reconstructed, according to traffic load, the attractor is shown in Fig. 3. 


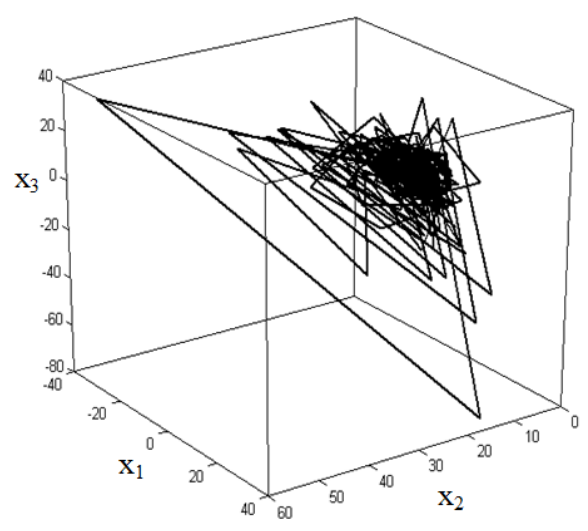

Fig. 3. Attractor, built on the basis of network traffic

In general, studies of chaotic signal can be formulated as follows.

1) Construction of the histogram. If there is heavy-tailed, it is necessary to check the chaos.

2) Calculation of the necessary conditions - Lyapunov exponent, Hurst exponent.

3) Construction of the attractor and the identification of symmetry breaking.

If all three tests are accomplished, there is a chaos in the system, and this property should be considered when dealing with such networks.

Confirmation of chaos can be the basis for building dynamic models. For example, in the form of an ensemble of pendulums [1], affinity controlled systems [8] or in the form of rows.

Identification of the parameters of the system using the method of $[3,8]$, gives the following result:

$$
\begin{gathered}
d \mathbf{x} / d t=\mathbf{A x}(t)+\Psi_{0}(t) \\
A=\left[\begin{array}{rrrr}
0.9413 & -0.1805 & 0.1164 & -0.0295 \\
-0.0545 & 0.8226 & 0.1622 & 0.1056 \\
0.0014 & -0.0105 & -0.4455 & 0.8474 \\
-0.0062 & 0.0341 & -0.8860 & -0.5404
\end{array}\right] \\
\Psi_{0}=\left[\begin{array}{l}
0.0399 \\
0.0463 \\
-0.4848 \\
-0.1851
\end{array}\right]\left(\exp \left(t^{0.0001}\right) \sin \left(t^{0.4}\right)\right) .
\end{gathered}
$$

\section{CONCLUSION}

The paper deals with the chaotic phenomena in computer data networks. Based on the chaotic properties can be constructed mathematical models of the dynamic behavior of traffic. Models can be used to provide guaranteed quality of service (QoS), the analysis of bottlenecks in the structure of the corporate network, data sharing in cloud environments $[9,10]$.

At the same time, and the chaos of the indicators themselves, the structure of the attractor can have the value. Changing the values of the highest Lyapunov exponent, topology change attractor is an indicator of changes in network activity. For example, computer attacks $[6,10]$, the failure (denial of service) enterprise data exchange, or a reason for the change of policy administration - an extension of communication channels or by completing a list of banned network resources. For example, this is the case of recently observed popularity of social networking, video sharing resources.

\section{REFERENCES}

[1] A. V. Karpukhin, I. N. Kudryavtsev, A. V. Borisov, D. I. Gritsiv and H. Cho "Computer Simulation of Chaotic Phenomena in High-Speed Communication Networks," Journal of Korean Institute of Information Technology, 2013, vol. 11, no. 3, pp. 113-122.

[2] Z. Liu "Chaotic Time Series Analysis," Mathematical Problems in Engineering, 2010, vol. 2010.. (doi:10.1155/2010/720190)

[3] E. V. Nikulchev and O. V. Kozlov "Identification of Structural Model for Chaotic Systems," Journal of Modern Physics, 2013, vol. 4, no. 10, pp. 1381-1392. (doi: 10.4236/jmp.2013.410166)

[4] W. Xiong, H. Hu, N. Xiong, L. T. Yang, W. C. Peng, X. Wang, Y. Qu "Anomaly secure detection methods by analyzing dynamic characteristics of the network traffic in cloud communications," Information Sciences, 2014, vol. 258, pp. 403-415. (doi: 10.1016/j.ins.2013.04.009).

[5] R. O. Grigoriev "Identification and Control of Symmetric Systems," Physica D, 2000, vol. 140, no.3-4, pp. 171-192. (doi: 10.1016/S01672789(00)00014-2).

[6] P. Chossat and M. Golubitsky "Symmetry-increasing bifurcation of chaotic attractors," Physica D: Nonlinear Phenomena, 1988, vol. 32, no. 3, pp. 423-436.

[7] E. V. Nikulchev "Geometric method of reconstructing systems from experimental data," Technical Physics Letters, 2007, vol. 33, no. 3, pp. 267-269. (doi: 10.1134/S1063785007030248)

[8] E. V. Nikulchev "Geometric Method of Reconstructing Evolution Equations from Experimental Data," in Evolution Equations, A. L. Claes, Eds. New York : Nova Science Publishers, 2011, pp. 373-379.

[9] E. V. Pluzhnik and E. V. Nikulchev "Use of dynamical systems modeling to hybrid cloud database," Int'1 J. of Communications, Network and System Sciences, 2013, vol. 6, no. 12, pp. 505-512. (doi: 10.4236/ijens.2013.612054)

[10] E. Pluzhnik, E. Nikulchev and S. Payain "Laboratory Test Bench for Research Network and Cloud Computing" Int'1 J. of Communications, Network and System Sciences, 2014, vol. 7, no.7, pp. 243-247. (doi: 10.4236/ijcns.2014.77026) 